\title{
Normes et marges
}

Coleen EVEN, Université de Waterloo

Krysteena GADZALA, Université de Waterloo

Avec le dossier Norme $(s)$ et Marge( $s)$ nous tenons à partager les articles préparés dans le cadre du colloque «Convergences» des étudiants d'études supérieures en français tenu à Waterloo en avril 2016. Le projet de ce dossier remonte à une initiative des étudiants en études supérieures en français de l'Université de Waterloo en collaboration avec Catherine Parayre de l’Université Brock. Lors de l'assemblée générale de l'APFUCC (Association des professeur.e.s de français des universités et collèges canadiens) au cours du Congrès de la Fédération canadienne des sciences humaines de 2015, nous sommes entrées en contact avec Catherine Parayre dans le but de publier les actes du colloque annuel de l'AEESF (Association des étudiants d'études supérieures en français) dans la revue Voix plurielles. Le colloque réunit chaque année des étudiants de multiples universités canadiennes et a récemment pris de l'envergure grâce aux contributions d'étudiants internationaux. Nous souhaitions ainsi partager ces nombreuses communications originales et enrichissantes avec la communauté scientifique.

Dans ce dossier, les articles abordent la problématique du colloque à travers de multiples formats, notamment des récits de voyage, des romans, des pièces de théâtre, des peintures, des romans de fantasy pour jeunesse et un recueil de nouvelles. Eric D’Avernas reprend le corpus de Samuel Champlain afin d'examiner l'œuvre d'après la théorie écocritique. Son étude porte sur l'imposition de la norme européenne au Canada au dix-septième siècle, à l'époque où l'Europe se forge une image de l'espace des Grandes découvertes. L'article se sert en particulier de l'iconographie afin de mieux illustrer l'établissement de cette norme à propos des territoires autochtones et souligne la marginalisation qui en ressort. Ariane Bellemare propose une étude du public à partir de la pièce Trois de Mani Soleymanlou. Son travail aborde la question de l'origine culturelle et de la réception théâtrale. L'étude, qui se sert du concept d'intraculturalité, porte sur la réaction du public afin de comprendre si l'échange représenté sur scène permet aux spectateurs d'aborder la question de l'immigration, de l'autre et de la diversité de manière ouverte. Tina Chan dans son article «Cœur d'animal : l'emploi des animaux pour établir le portrait de l'animalité chez les humains dans les œuvres littéraires et picturales à travers les 
âges » revient sur la place des animaux par rapport à la construction des qualités et des caractéristiques des êtres humains en analysant une série de tableaux de Rubens et d'Alex Colville ainsi que d'ouvrages littéraires tels que le roman La rage (1995) de l'écrivain québécois Louis Hamelin, de la pièce de théâtre québécois Tom à la ferme (2011) de Michel Marc Bouchard et du recueil de nouvelles intitulé La héronnière (2003) de Lise Tremblay. Camille Contré présente une analyse sur le physique de la transgression dans La trilogie de Mallaig. Son travail examine la déformation physique chez des personnages monstrueux de la trilogie tels que ceux atteints d'une difformité, d'une maladie ou d'une blessure et revient sur la marginalisation qui les affecte.

Prenant comme exemple le personnage principal de Celaena Sardothian dans la série Throne of Glass de Sarah J. Maas, Fanie Demeule étudie l'identité et la représentation picturale ainsi que leurs effets sur le lectorat. Son article déconstruit l'identité d'une guerrière dont le rapport à la violence influence son image qui oscille entre des traits féminins et masculins. Maryse Sullivan quant à elle examine l'œuvre de Maryse Condé, Moi, Tituba sorcière... noire de Salem pour proposer une lecture et une analyse sociocritique de l'ouvrage afin de mettre en relief la manipulation de la figure de la sorcière et de la femme, souvent marginalisées. L'examen conduit au sein de l'article présente la réhabilitation effectuée dans l'œuvre de l'auteure.

La thématique du colloque permet d'aborder des œuvres de multiples horizons grâce à de nouvelles approches ou selon des perspectives originales. De l'écocritique à l'étude de public, il s'agit d'explorer les notions de normes et de marge vis-à-vis des personnages, du récit ou de la relation entre le lecteur (voir le spectateur) et l'œuvre. Nous vous souhaitons ainsi une très bonne lecture. 